\title{
Krüppel-like factor 8 induces epithelial-to-mesenchymal transition and promotes invasion of pancreatic cancer cells through transcriptional activation of four and a half LIM-only protein 2
}

\author{
XIAOPING YI ${ }^{1,2 *}$, HONGYAN ZAI ${ }^{3 *}$, XUEYING LONG ${ }^{1}$, XIAOYI WANG ${ }^{1}$, WENZHENG LI ${ }^{1}$ and YIXIONG LI $^{3}$ \\ ${ }^{1}$ Department of Radiology, ${ }^{2}$ Postdoctoral Research Workstation of Pathology and Pathophysiology and ${ }^{3}$ Department of \\ General Surgery, Basic Medical Sciences, Xiangya Hospital, Central South University, Changsha, Hunan 410008, P.R. China
}

Received November 17, 2015; Accepted May 18, 2017

DOI: 10.3892/ol.2017.6734

\begin{abstract}
Pancreatic cancer (PC) is one of the most aggressive types of cancer with an extremely poor prognosis. Invasive growth and early metastasis is one of the greatest challenges to overcome for the treatment of PC. Numerous previous studies have indicated that the transcription factor Krüppel-like factor 8 (KLF8) and nuclear cofactor four and a half LIM-only protein 2 (FHL2) serve important roles in tumorigenesis and tumor progression; however, their roles in $\mathrm{PC}$ remain elusive. The present study revealed that KLF8 and FHL2 expression is aberrantly co-overexpressed in PC tissue samples and associated with tumor metastasis. Furthermore, a positive correlation between the expression levels of KLF8 and FHL2 was observed. Subsequently, the present study identified KLF8 as a critical inducer of epithelial-to-mesenchymal transition (EMT) and invasion. Of note, the present study demonstrated that KLF8 overexpression induced a strong increase in FHL2 expression, and subsequent promoter reporter assays determined that KLF8 directly bound and activated the FHL2 gene promoter. Furthermore, FHL2 knockdown in KLF8-overexpressing cells partially reversed the EMT and invasive phenotypes. The present study identified KLF8-induced FHL2 activation as a novel and critical signaling mechanism underlying human PC invasion.
\end{abstract}

\section{Introduction}

Pancreatic cancer (PC) is one of the most aggressive types of malignancy worldwide with notably poor prognosis (1) It is

Correspondence to: $\mathrm{Dr}$ Xiaoping $\mathrm{Yi}$ or Dr Wenzheng $\mathrm{Li}$, Department of Radiology, Xiangya Hospital, Central South University, 87 Xiangya Road, Changsha, Hunan 410008, P.R. China E-mail: doctoryixiaoping@126.com

E-mail: doctorwenzhengli@126.com

*Contributed equally

Key words: Krüppel-like factor 8, four and a half LIM-only protein 2, pancreatic cancer, epithelial-to-mesanchymal transition, invasion characterized by invasive growth and early metastasis, and is also highly resistant to chemotherapy and radiation therapy (2). Surgical resection remains the only potentially curative treatment for local patients with PC. However, $>85 \%$ of PC cases are diagnosed at an advanced stage. Even following radical surgical resection, the patients usually succumb to recurrence or metastasis after a short period of time (3). Therefore, PC represents one of the major challenges in oncology to date. It is necessary to investigate the mechanisms involved in PC in order to develop an effective treatment strategy.

Increasing evidence has highlighted the significance of Krüppel-like factor 8 (KLF8) in tumor progression and suggested that it may be a promising target for cancer treatment $(4,5)$. As a GT-box (CACCC) binding dual-transcription factor, KLF8 regulates the transcription of numerous genes by recruiting the $\mathrm{C}$-terminal binding protein $(\mathrm{CtBP})$ corepressor (6-10) or $\mathrm{p} 300$ and $\mathrm{P} 300 / \mathrm{CtBP}$-associated factor histone acetyltransferase co-activators $(8,11-14)$ in order to target gene promoters. It is aberrantly overexpressed in a number of cancer subtypes and has been implicated in the initiation, development and progression of these cancer subtypes, including hepatocellular carcinoma (15-17), gastric cancer (18-21), breast cancer $(22,23)$, ovarian cancer $(14,24)$, renal cancer $(22,25)$ and glioma (26). It is worth noting that KLF8 induced tumor cell epithelial-to-mesenchymal transition (EMT) and maintained the invasive potential of cancer, which appeared to serve a critical role in the metastatic progression of cancer cells $(6,27)$. Various target genes of KLF8 and signaling pathways associated with cancer have been identified (14,22,27-29). However, the role of KLF8 in PC remains to be elucidated and less is known about the mechanisms underlying KLF8-modulated expression of its target genes.

Four and a half LIM-only protein 2 (FHL2) is a member of the FHL family of proteins, which functions as a coactivator of numerous transcription factors, including $\beta$-catenin $(30,31)$. It is expressed in a cell- and tissue-specific manner, and contributes to the regulation of important cell functions, including cell adhesion, cell transcription and signal transduction (32-36). It has been reported that FHL2 was overexpressed in PC tissue samples and critically involved in cell survival, radio-resistance, proliferation and apoptosis in PC cells (32). FHL2 directly interacts with Snail2 and 
downregulates E-cadherin transcriptional activity, thereby inducing colorectal cancer cell EMT and invasion $(27,37,38)$. The upregulated expression level of FHL2 may be a trigger or mediator of EMT in cancer cells and is essential to maintain their malignant phenotype. Previous studies have indicated that FHL2 and KLF8 were overexpressed in numerous types of cancer, including colorectal cancer $(27)$, PC $(39,40)$, breast cancer $(36,41)$ and ovarian cancer $(42,43)$. It is plausible to consider that they may have a synergistic effect on cellular processes in association with malignancy. Notably, it was suggested in a more recent study that KLF8 induced FHL2-mediated EMT and potentially promoted colorectal cancer cell metastasis (27). However, confirmation of its role, and the possible mechanisms underlying the role KLF8 may serve in FHL2-mediated cell EMT, invasion and metastasis have not yet been investigated.

The present study firstly demonstrated that aberrant co-overexpression of KLF8 and FHL2 was associated with PC metastasis. Subsequently, the present study revealed that exogenous overexpression of KLF8 in PC cells induced cell EMT, and invasive and metastatic phenotypes. Furthermore, the present study determined that FHL2 was a direct target for transcriptional activation by KLF8, and FHL2 knockdown by short interfering RNA (siRNA) may partially reverse these effects induced by overexpressed KLF8. These results provided evidence supporting an important role of FHL2 downstream of KLF8 in promoting PC cell EMT and invasion. The results of the present study further supported a potentially important role that KLF8 serves in human PC development.

\section{Materials and methods}

Reagents and antibodies. The following antibodies and reagents were used: Anti-E-cadherin (cat. no. sc-8426), anti-Slug (cat. no. sc-166476; both Santa Cruz Biotechnology, Inc., Dallas, TX, USA), anti-FHL2 (cat. no. SAB2500398), anti-GAPDH (cat. no. G8795; Sigma-Aldrich; Merck KGaA, Darmstadt, Germany), Boyden Chambers, polycarbonate membranes (8- $\mu \mathrm{m}$ pore size; Neuro Probe, Inc., Gaithersburg, MD, USA), lipofectamine and oligofectamine (Invitrogen; Thermo Fisher Scientific, Inc., Waltham, MA, USA). The human Panc-1, SW1990, Capan-1, Bxpc-3 and Miapaca-2 PC cell lines were obtained from the American Type Culture Collection (Manassas, VA, USA) and cultured as previously described $(32,44)$.

Tissue samples. The present study was approved by the Ethics Committee of Xiangya Hospital, Central Southern University (Changsha, China; no. 201406373). Written informed consent was obtained from all the patients prior to enrollment in the present study. A total of 34 pairs of surgically resected specimens, including tumor tissue samples and their noncancerous counterparts, were obtained at the Department of General Surgery, Xiangya Hospital between September 2010 and June 2015. The patients included 20 males and 14 females, with a mean age of 56 (range, 33-76) years. Among them, 18 patients did not exhibit lymph node metastasis, with a male to female ratio of 8:10 and a mean age of 58.4 (range, 35-75) years. The remaining 16 patients presented with lymph node metastasis, with a male to female ratio of 9:7 and a mean age of 53.4 (range, 33-76) years.

Reverse transcription-quantitative polymerase chain reaction (RT-qPCR). Total RNA extraction and RT-qPCR were performed as previously described $(32,44)$. The primer sequences used were as follows: KLF8 sense, 5'-TTCAGA AGGTGGCTCAATGC-3' and KLF8 antisense, 5'-GGAGTG TTGGAGAAGTCATATTAC-3'; FHL2 sense, 5'-TCCATA CTGCCTGACCTGC-3' and FHL2 antisense, 5'-TTGGCG TTCCTCGAAAGAG-3'; and GAPDH sense, 5'-TGACTT CAACAGCGACACCCA-3' and GAPDH antisense, 5'-CAC CCTGTTGCTGTAGCCAAA-3'. For each sample, triplicate determinations were performed, and mean values were used for further calculations, with GAPDH detected as the internal control. The relative quantitative expression of the target gene compared with that of GAPDH using the $2^{-\Delta \Delta \mathrm{Cq}}$ method (45).

Western blotting (WB). WB was performed as described previously (44). Briefly, pancreatic cells (including Panc-1, Sw1990, Bxpc-3, Capan-1, Miapaca-2) were harvested and lysed in lysis buffer. Subsequently, total protein $(50 \mu \mathrm{g})$ was subjected to $10 \%$ SDS-PAGE and transferred onto polyvinylidene fluoride membranes (EMD Millipore, Billerica, MA, USA). Primary antibodies were diluted according to the manufacturer's recommendations. The relative content of each protein was detected by enhanced chemiluminescence (GE Healthcare, Chicago, IL, USA). Endogenous GAPDH was used as an internal control.

Cell transfection and clone selection. pcDNA3.1 vectors expressing KLF8 or mock messenger RNA (mRNA) were constructed, as previously described (17). KLF8 or mock vectors were transfected into Panc-1 cells using Lipofectamine 2000 reagent (Invitrogen; Thermo Fisher Scientific, Inc.). The transfected cells were screened with $400 \mu \mathrm{g} / \mathrm{ml} \mathrm{G} 418$ (Merck $\mathrm{KGaA}$ ) for 4-6 weeks. Clones of stably transected cells were obtained by the limited dilution method, and the overexpression efficiency was confirmed by WB. Transfections of FHL2 siRNA (5'-GCCAAUUGGAACCAAGAGUTT-3') were performed as previously described (32). The scrambled (scr) siRNA (5'-TTCTCCGAACGTGTCACGT-3') was used as the negative control. Following a 24-h incubation at $37^{\circ} \mathrm{C}$, cells were used for further experiments. The knockdown efficiency was detected by WB.

Cell invasion analysis. An invasion assay was performed as previously described (46). Transwell polycarbonate filters (8- $\mu \mathrm{m}$ pore size; Corning Incorporated, Corning, NY, USA) were used. A total of $2 \times 10^{5}$ Panc- 1 cells were plated in the top chamber of the Transwell with a Matrigel-coated polycarbonate membrane. The bottom wells of the system were filled with $10 \%$ fetal bovine serum complete medium (Gibco; Thermo Fisher Scientific). Following a 24 -h incubation at $37^{\circ} \mathrm{C}$, the number of transmigrated cells was counted, as previously described (46).

Promoter reporter and dual luciferase assays. For the validation of FHL2 as a direct target of KLF8, a luciferase reporter assay was performed. Briefly, 55-basepair fragments of the 
FHL2 promoter upstream of the transcription start site were cloned into the pGL3 basic vector (Promega Corporation, Madison, WI, USA), and the pLuc55 mutant construct was created by site-directed mutagenesis of the FHL2 promoter vector (Stratagene; Agilent Technologies, Inc., Santa Clara, CA, USA), as previously described (27). The primer sequences were listed as follows: pLuc55 forward (KpnI), 5'-GGGGTA CCGGGGGGTGCACAGAGGTGGAGC-3' and reverse $(X h o \mathrm{I})$, 5'-TCCGCTCGAGCGAGGCCTCATATTTTC CAG-3'; and pLuc55-MT (site-directed mutagenesis) forward, 5'-CCTAATCTGGGGAGTACACAGAGGTGGAGCTGAGCA GCC-3' and reverse, 5'-GGCTGCTCAGCTCCACCTCTG TGTACTCCCCAGATTAGG-3'. Wild-type or mutant reporter constructs were co-transfected with Lipofectamine 2000 transfection reagent (Invitrogen; Thermo Fisher Scientific, Inc.) into the Panc- 1 cells with KLF8 or mock vectors, according to the manufacturer's protocol. After a $48 \mathrm{~h}$ incubation at $37^{\circ} \mathrm{C}$, the luciferase activity was evaluated with the Dual-Luciferase ${ }^{\circledR}$ Reporter Assay System (Promega Corporation). The relative luciferase activity was normalized to that of firefly luciferase. The transcriptional activity at the promoter was presented as the fold change of relative luciferase units compared with those of the basic pGL3 vector control.

Statistical analysis. Data are presented as the mean \pm standard deviation. The comparison between the test and control group was evaluated by Student's t-test. All statistical analyses were performed using SPSS version 15.0 software (SPSS, Inc., Chicago, IL, USA). P $<0.05$ was considered to indicate a statistically significant difference. Assays were performed $\geq 3$ times with triplicate samples independently.

\section{Results}

Aberrant co-overexpression of KLF8 and FHL2 in PC is associated with tumor metastasis. In order to investigate the association between aberrant expression of KLF8 and FHL2 in PC, RT-qPCR and WB were performed to detect the expression levels of these genes and proteins, respectively, in 34 pairs of cancer tissues and adjacent normal pancreatic tissues. Of note, patients with PC and lymph node metastasis exhibited higher KLF8 and FHL2 mRNA expression levels compared with those exhibited by patients with PC without lymph node metastasis $(\mathrm{P}<0.01$; Fig. $1 \mathrm{~A}$ and $\mathrm{B})$. Furthermore, KLF8 mRNA was positively correlated with FHL2 mRNA expression level $\left(\mathrm{r}^{2}=0.673 \mathrm{P}<0.001\right)$ in $\mathrm{PC}$ tissue samples $(\mathrm{P}<0.01$, Fig. 1D). Additionally, relative higher protein expression levels of KLF8 and FHL2 were also observed in the resected tumor tissue samples with lymph node metastasis in comparison with those in tissue samples without lymph node metastasis, as determined by WB (Fig. 1E).

In order to validate our findings in vitro, the expression levels of KLF8 and FHL2 protein were investigated in five PC cell lines: Panc-1, Capan-1, Bxpc-3, Miapaca-2 and SW1990. As expected, the expression levels of KLF8 and FHL2 were positively associated with the metastatic potential of the PC cell lines. As presented in Fig. 1C, KLF8 was expressed at a relatively high level in Panc-1, Capan-1, SW1990 and Bxpc-3 cells, and at a relatively low level in Miapaca- 2 cells. The FHL2 expression level was similar to that of KLF8 in the five cell lines, with the exception of SW1990 cells. Panc-1 cells, with the highest metastatic potential in the five aforementioned cell lines (47), expressed significantly higher expression levels of KLF8 compared with those expressed by the other PC cell lines. These results indicated that aberrant co-overexpression of KLF8 and FHL2 was associated with tumor metastasis in PC.

KLF8 upregulates FHL2 expression levels to induce EMT and promote cell invasion in a human PC cell line. In order to investigate the biological role of KLF8 in PC, the present study first determined whether KLF8 was sufficient to promote invasion using exogenous introduction of KLF8 in Panc-1 cells. Overexpression of KLF8 protein in the stably transfected cells was confirmed by WB (Fig. 2A).

In order to investigate the effect of KLF8 on EMT induction, morphological examination was conducted and the expression levels of EMT markers were analyzed. It was also demonstrated that overexpression of KLF8 in Panc-1 cells resulted in a shift in EMT marker expression levels by WB. Upregulation of mesenchymal markers (Slug) and downregulation of the epithelial marker E-cadherin were observed by WB in the stable vector transfectants. The results demonstrated that induction of KLF8 expression induced a significant increase in FHL2 protein expression (Fig. 2A). In addition, the present study revealed that exogenous induction of KLF8 expression resulted in a $\geq 3$-fold increase in cell invasiveness (Fig. 2B-D). The present study also investigated the morphological features of Panc-1 cells. The stable KLF8 transfectants exhibited spindle-like, fibroblastic morphology, which is one of the main characteristics of EMT (6). Dendritic-like or long cytoplasmic processes were observed under a phase-contrast microscope; however, the stable vector transfectants (control) presented a round or flat morphology with a short cytoplasmic process (Fig. 2E and F).

These results may suggest the effect of co-overexpression of FHL2 and KLF8 on EMT and cell invasion. Based on the in vitro overexpression experiments, KLF8 appeared to be a potent inducer of EMT.

FHL2 is required for KLF8-mediated EMT and invasive phenotypes. According to previous studies, FHL2 may induce tumor cell EMT and maintain the invasive phenotype of cancer cells (27); however, the role of FHL2 in KLF8-induced EMT in PC cells remains elusive. Downregulation of FHL2 expression by siRNA was performed in KLF8-overexpressing Panc-1 cells and resulted in the conversion from the expression of the mesenchymal marker Slug to the epithelial marker E-cadherin when compared with that of the control FHL2-scr siRNA cells (Fig. 2G). Cell invasion analysis indicated that the number of invasive cells in the FHL2-siRNA-treated group decreased significantly compared with those in the control group (Fig. 2H, I and J). Morphological examination demonstrated that FHL2 downregulation in KLF8-overexpressing cells induced partial reversal of the EMT process, i.e. the mesenchymal-to-epithelial transition process (Fig. $2 \mathrm{~K}$ and L). Taken together, a critical role for FHL2 on the EMT and invasion phenotypes induced by abnormal KLF8 expression in PC cells is indicated.

FHL2 is a direct transcriptional activation target of KLF8. The present study assessed whether the FHL2 promoter may be 
A

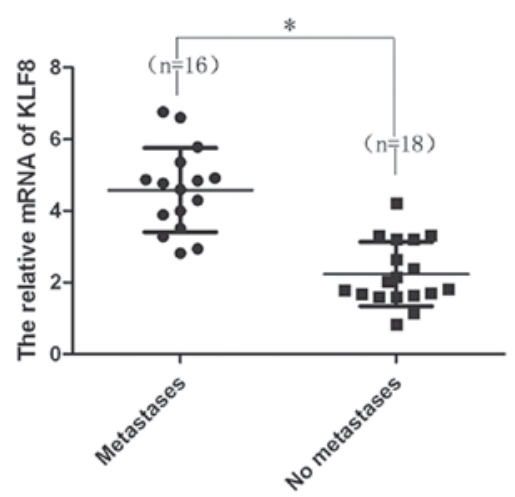

C

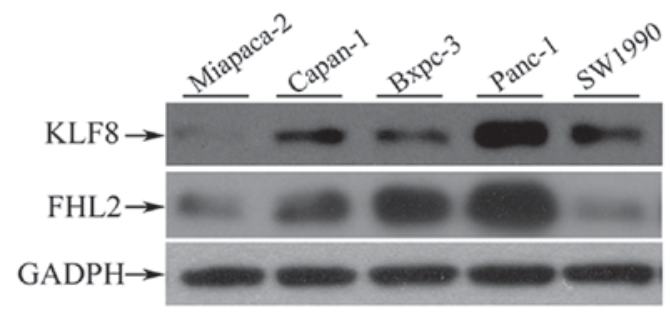

E
B

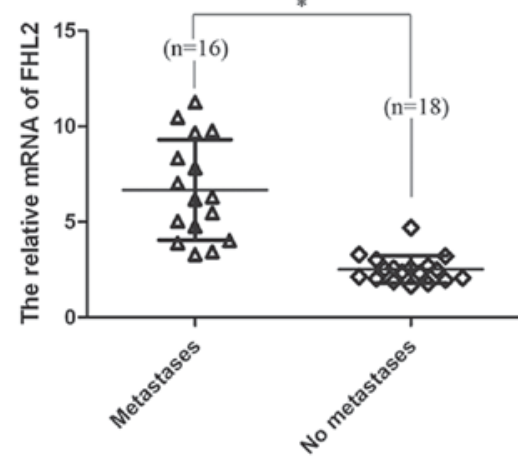

$\mathrm{D}$

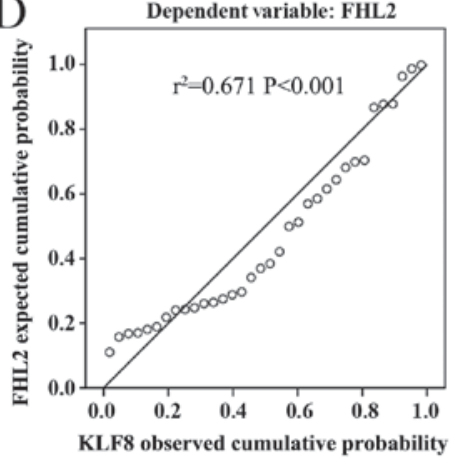

No metastasis

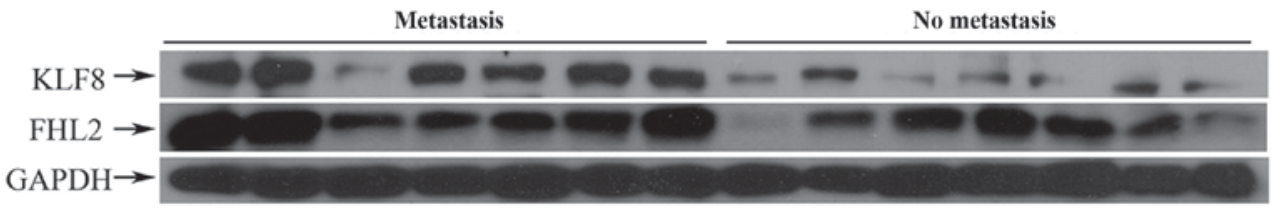

Figure 1. Expression levels of KLF8 and FHL2 in PC tissue samples and cell lines. Patients with PC with lymph node metastases exhibited higher (A) KLF8 and (B) FHL2 mRNA expression levels compared with those exhibited by patients without lymph node metastases ("P $<0.001)$. (C) WB analysis of KLF8 and FHL2 protein expression levels in pancreatic cell lines: Miapaca-2, Capan-1, Bxpc-3, Panc-1 and SW1990. GAPDH was used as the internal control. (D) The patients with high expression levels of KLF8 also demonstrated high FHL2 expression levels. (E) Protein expression levels of KLF8 and FHL2 in tumor samples of patients with PC with or without lymph node metastasis by WB analysis. GAPDH expression levels were used as internal controls. KLF8, Krüppel-like factor 8; FHL2, four and a half LIM-only protein 2; PC, pancreatic cancer; WB, western blot.

directly activated by the KLF8 protein using a dual-luciferase reporter system. The promoter regions containing the GT-box (-50 to -55$)$, which are the potential binding sites, of human FHL2 were cloned upstream of a luciferase gene in a reporter plasmid (Fig. 3A and B). Subsequently, transient transfections were performed to explore whether the FHL2 promoter was activated by overexpressed KLF8. The results revealed that the luciferase activity increased by $3.02 \pm 0.12$-fold compared with that of the vector control $(\mathrm{P}<0.001)$ (Fig. 3C). Correspondingly, the present study also mutated two nucleotides of the identified GT-box binding region to generate a FHL2 3'-untranslated region (UTR) mutant in the KLF8 target region to disrupt binding (Fig. 3B). Conversely, the increased effect of luciferase activity was not observed in cells carrying the mutant-type construct of the FHL2 3'-UTR (Fig. 3C). These results suggested that FHL2 may be a direct transcriptional activation target of KLF8, and the proximal GT-box at -55 is the main KLF8-binding site in the FHL2 promoter (Fig. 3).

\section{Discussion}

PC is known for its aggressive growth; mortality usually results from tissue invasion and early metastasis $(2,3)$. Similarly to the majority of other carcinoma types, $\mathrm{PC}$ progression is associated with EMT (48). It has been widely accepted that EMT frequently occurs in PC and is involved in tumorigenesis, cancer progression and metastasis (49). Therefore, identification of critical factors associated with tumor progression and EMT, and their underlying mechanisms are particularly important.

Loss of expression of E-cadherin, an epithelial marker, is a hallmark of EMT $(6,48,50)$. An increasing number of transcription factors have been implicated in the repression of E-cadherin expression, including KLF transcription factor family proteins $(6,23)$. KLF8 is a relatively new member of this family and is emerging as a crucial regulator of cancer initiation and progression (10). With accumulating evidence demonstrating its overexpression in numerous types of aggressive human cancer and its vital role during a variety of cellular processes in cancer, KLF8 has become a novel focus in cancer research and a potential target for the treatment of cancer $(4,5)$. Notably, a number of previous studies have revealed that KLF8 induces tumor cell EMT and maintains the invasive potential of cancer, which serves a critical role in the regulation of various cancer-associated cellular processes favoring tumor metastatic progression $(6,23,24)$. Our previous study demonstrated that 


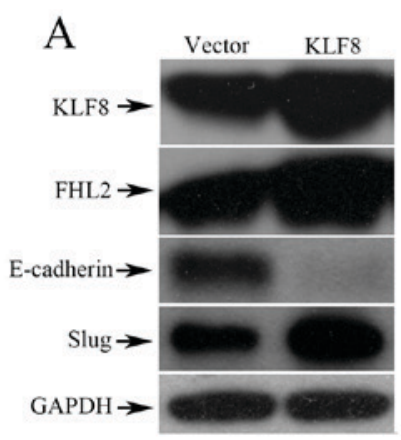

B

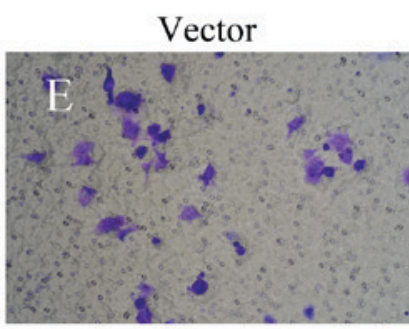

Vector

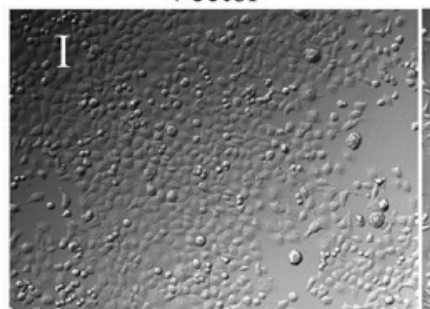

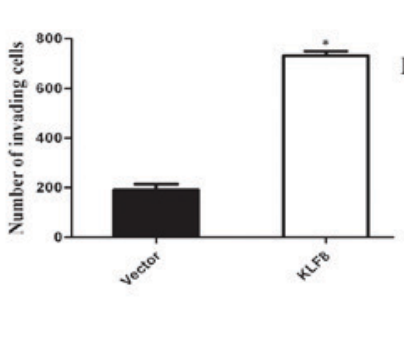

KLF8

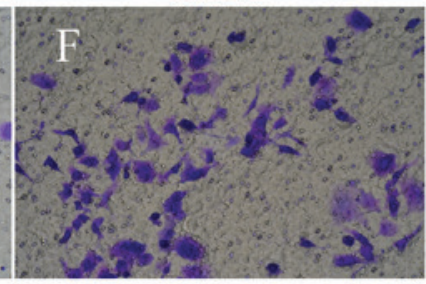

KLF8
$\mathrm{C}$

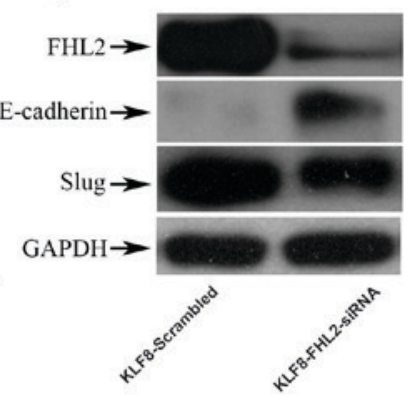

KLF8-Scr
$\mathrm{D}$

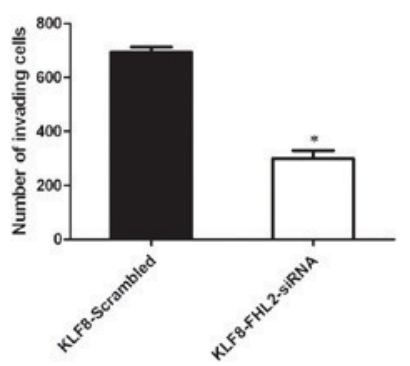

KLF8-FHL2-siRNA

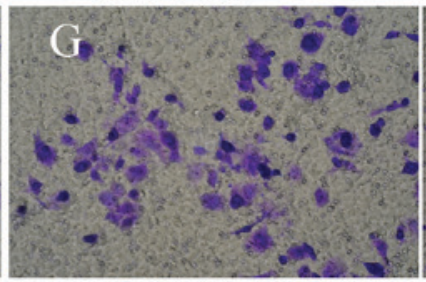

KLF8-Scr siRNA

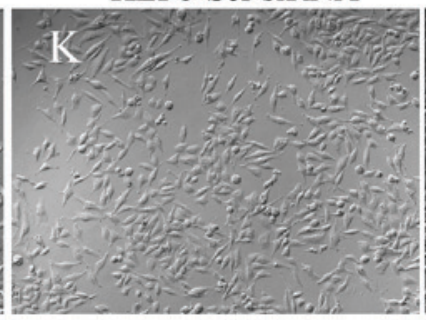

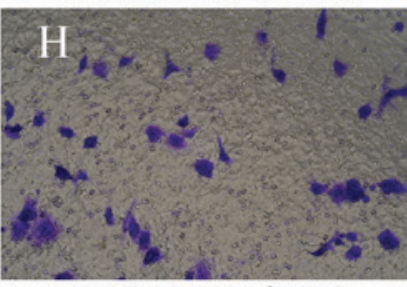

KLF8-FHL2-siRNA

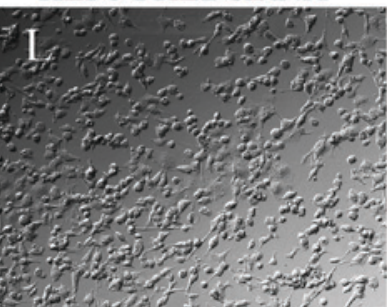

Figure 2. KLF8 upregulates FHL2 expression to induce EMT and promote cell invasion. (A) KLF8, FHL2, E-cadherin and Slug expression levels in stable KLF8 transfectants in Panc-1 cells were detected by WB. GAPDH was used as the internal control. (B) Quantification of an invasion assay performed with or without KLF8 transfection. (C) Expression of epithelial-to-mesenchymal transition-associated biomarkers, including E-cadherin, Slug and FHL2, were detected by WB $72 \mathrm{~h}$ following transfection with KLF8-scr or KLF8-FHL2 siRNA. (D) Quantification of an invasion assay performed with KLF8-scr or KLF8-FHL2 siRNA. Invasive potential of Panc-1 stable transfectants following transfection with (E) vector, (F) KLF8, (G) KLF8-scr siRNA and (H) KLF8-FHL siNRA. Morphology of Panc-1 cells transfected with (I) vector, (J) KLF8, (K) KLF8-scr siRNA and (L) KLF8-FHL2-siRNA cells, visualized by phase-contrast microscopy. Magnification, $\mathrm{x} 100$. "P<0.05 vs. other group. KLF8, Krüppel-like factor 8; FHL2, four and a half LIM-only protein 2; EMT, epithelial-to-mesenchymal transition; scr, scrambled; siRNA, short interfering RNA; WB, western blotting.
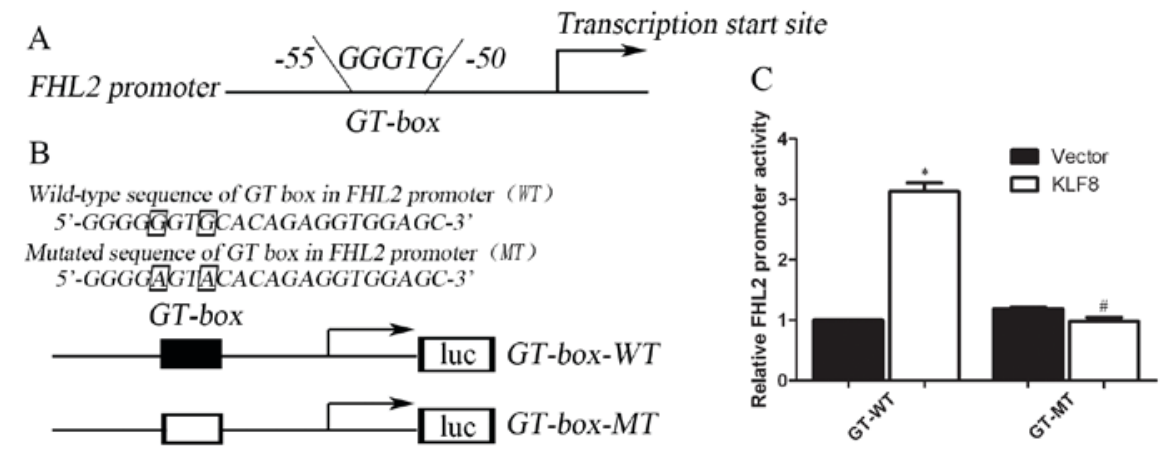

Figure 3. FHL2 is a direct transcriptional activation target of KLF8. (A) The luciferase reporter constructs contained the FHL2 promoter with potential KLF8 binding sites upstream of a luciferase gene. (B) Transcriptional activities of reporters in the transient transfected cells were detected using a dual-luciferase assay, and the results are expressed as fold-change of relative luciferase units. "P $<0.001 ;{ }^{*} \mathrm{P}>0.05$. (C) Primer sequences used for site-directed mutations. Site-directed mutagenesis of GT-box in pLuc55 was performed to generate mutations, and the mutated nucleotides were marked with square frames. WT, wild-type; MT, mutant; KLF8, Krüppel-like factor 8; FHL2, four and a half LIM-only protein 2; EMT, epithelial-to-mesenchymal transition; luc, luciferase.

KLF8 was upregulated in PC tissues and cell lines, which serves a critical role in PC cell proliferation by promoting the $\mathrm{G}_{2} / \mathrm{M}$ phase progression via up- or downregulating numerous cell cycle-associated proteins (50). However, to the best of our knowledge, whether the mechanisms underlying KLF8 serve a role in cancer cell invasion and metastasis has not previously been investigated.

The present study demonstrated that KLF8 was overexpressed in highly metastatic PC cell lines and in patients with lymphatic metastasis, which indicated that KLF8-positive 
tumor cells may have more aggressive phenotypes than KLF8-negative tumor cells. Based on the exogenous overexpression experiments in vitro, KLF8 was revealed to be a potent inducer of EMT. In agreement with our finding, a number of previous studies have demonstrated that ectopic expression of KLF8 repressed E-cadherin expression, induced tumor cell EMT, and increased cell invasion and metastasis in breast cancer $(6,22)$ and hepatocellular carcinoma (17). Furthermore, downregulation of KLF8 may inhibit cell invasion and metastasis in gastric cancer $(23,24)$.

Of note, the present study identified the KLF8-mediated upregulation of FHL2 as a novel mechanism for induction of EMT and promotion of invasion in human PC cells. Firstly, the present study identified a strong association between the co-expression of KLF8 and FHL2 in PC tumors with lymph node metastasis. Secondly, the present study demonstrated that KLF8 expression promotes FHL2 transcription in human PC cells. Finally, the present study provided evidence that FHL2 was required for KLF8-induced EMT and metastatic phenotypes in vitro. These results suggested a potentially significant role for the cooperative association between FHL2 and KLF8 in promoting PC invasion and metastasis, and may shed new light on the underlying molecular mechanisms.

It is known that FHL2 is aberrantly upregulated in invasive PC tissues (32), as revealed in present study. Notably, the present study identified FHL2 as a novel target of transcriptional activation by KLF8, which was demonstrated by a dual-luciferase reporter system that identified that KLF8 directly binds to the FHL2 promoter at the GT-box ( -50 to -55$)$. Of note, previous studies have revealed that KLF8 directly bound to a GT-box located in the E-cadherin promoter, repressed its expression and triggered the subsequent EMT process in the MDCK canine kidney epithelial cell line, and in the MCF-10A and MDA-MB-231 human mammary epithelial cell lines $(6,51)$. In addition, this process does not appear to depend upon other EMT inducer proteins such as Snail. This may explain why the EMT phenotype of KLF8-overexpressing PC cells was partially reversed following FHL2 knockdown. KLF8-associated target genes involved in EMT and how KLF8 regulates the EMT process require further investigation.

The present study demonstrated that FHL2 was highly overexpressed in PC tumor tissues, and suggested that FHL2 was involved in KLF8-induced EMT and invasion in vitro; however, the molecular mechanisms underlying the aberrant high expression level of FHL2 in PC, and whether and how FHL2 serves a role in vivo for the progression of $\mathrm{PC}$ require further elucidation. Previous studies have revealed that FHL2 was overexpressed in PC and served an important role in the survival and radiosensitivity of PC cell lines grown using three-dimensional cultures of laminin-rich extracellular matrix (32). However, the role of FHL2 in cancer remains ambivalent because FHL2 binds to various proteins with distinct functions (31). The roles of KLF8 and FHL2 in PC are unknown. The present study demonstrated that FHL2 protein expression level was highly associated with KLF8 expression level in PC tumor tissues with lymph node metastasis. Furthermore, FHL2 repression resulted in a marked reversion of the KLF8-induced cell EMT phenotype in vitro. Therefore, the present study suggested that KLF8 and FHL2 may cooperatively exert roles in PC progression. Further experiments are required to investigate the detailed mechanism.
In conclusion, the present study identified KLF8/FHL2 as a novel signaling pathway responsible for EMT and invasion in PC cells, and opened a new avenue in the research of KLF8 and FHL2. Notably, the expression level of KLF8 is barely detectable in normal epithelial cells (6), and in addition to FHL2, KLF8 also targeted E-cadherin and other factors associated with invasion and metastasis. Therefore, KLF8 may represent a novel promising target for intervention against PC cell invasion and metastasis.

\section{Acknowledgements}

The present study was supported by The Freedom Exploration Program of Central South University (grant no. 2011QNZT153), Hunan Provincial Natural Science Foundation of China (grant no. 14JJ6001) and The Foundation of Science and Technology Department of Hunan Province (grant no. 2014SK3268). Dr Xiaoping Yi is a Postdoctoral Fellow in Postdoctoral Research Workstation of Pathology and Pathophysiology, Basic Medical Sciences, Xiangya Hospital, Central South University. The authors thank all the members of the laboratory of Yixiong Li for the useful discussions and technical assistance provided.

\section{References}

1. Siegel RL, Miller KD and Jemal A: Cancer statistics, 2015. CA Cancer J Clin 65: 5-29, 2015.

2. Garrido-Laguna I and Hidalgo M: Pancreatic cancer: From state-of-the-art treatments to promising novel therapies. Nat Rev Clin Oncol 12: 319-334, 2015.

3. Hartwig W, Werner J, Jäger D, Debus J and Büchler MW: Improvement of surgical results for pancreatic cancer. Lancet Oncol 14: e476-e485, 2013.

4. Lu XJ, Shi Y, Chen JL and Ma S: Krüppel-like factors in hepatocellular carcinoma. Tumour Biol 36: 533-541, 2015.

5. Lahiri SK and Zhao J: Krüppel-like factor 8 emerges as an important regulator of cancer. Am J Transl Res 4: 357-363, 2012.

6. Wang X, Zheng M, Liu G, Xia W, McKeown-Longo PJ, Hung MC and Zhao J: Krüppel-like factor 8 induces epithelial to mesenchymal transition and epithelial cell invasion. Cancer Res 67: 7184-7193, 2007.

7. Hu JH, Navas P, Cao H, Stamatoyannopoulos G and Song CZ: Systematic RNAi studies on the role of Sp/KLF factors in globin gene expression and erythroid differentiation. J Mol Biol 366: 1064-1073, 2007.

8. Wei H, Wang X, Gan B, Urvalek AM, Melkoumian ZK, Guan JL and Zhao J: Sumoylation delimits KLF8 transcriptional activity associated with the cell cycle regulation. J Biol Chem 281: 16664-16671, 2006.

9. Zhang P, Basu P, Redmond LC, Morris PE, Rupon JW, Ginder GD and Lloyd JA: A functional screen for Krüppel-like factors that regulate the human gamma-globin gene through the CACCC promoter element. Blood Cells Mol Dis 35: 227-235, 2005.

10. van Vliet J, Turner J and Crossley M: Human Krüppel-like factor 8: A CACCC-box binding protein that associates with CtBP and represses transcription. Nucleic Acids Res 28: 1955-1962, 2000.

11. Lloyd JA: KLF8 sets the pace for the cell cycle through interactions with p300 and PCAF. Cell Cycle 9: 650-651, 2010.

12. Urvalek AM, Wang X, Lu H and Zhao J: KLF8 recruits the p300 and PCAF co-activators to its amino terminal activation domain to activate transcription. Cell Cycle 9: 601-611, 2010.

13. Mehta TS, Lu H, Wang X, Urvalek AM, Nguyen KH, Monzur F, Hammond JD, Ma JQ and Zhao J: A unique sequence in the $\mathrm{N}$-terminal regulatory region controls the nuclear localization of KLF8 by cooperating with the C-terminal zinc-fingers. Cell Res 19: 1098-1109,2009.

14. Zhao J, Bian ZC, Yee K, Chen BP, Chien S and Guan JL: Identification of transcription factor KLF8 as a downstream target of focal adhesion kinase in its regulation of cyclin D1 and cell cycle progression. Mol Cell 11: 1503-1515, 2003. 
15. Yang T, Cai SY, Zhang J, Lu JH, Lin C, Zhai J, Wu MC and Shen F: Krüppel-like factor 8 is a new Wnt/beta-catenin signaling target gene and regulator in hepatocellular carcinoma. PLoS One 7: e39668, 2012.

16. Han S, Han L, Sun H, Zan X, Zhou Z, Xu K, Yao Y and Liu Q: Krüppel-like factor expression and correlation with FAK, MMP9 and E-cadherin expression in hepatocellular carcinoma. Mol Med Rep 8: 81-88, 2013.

17. Li JC, Yang XR, Sun HX, Xu Y, Zhou J, Qiu SJ, Ke AW, Cui YH, Wang ZJ, Wang WM, et al: Up-regulation of Krüppel-like factor 8 promotes tumor invasion and indicates poor prognosis for hepatocellular carcinoma. Gastroenterology 139: 2146-2157.e12, 2010.

18. Chen G, Yang W, Jin W, Wang Y, Tao C and Yu Z: Lentivirus-mediated gene silencing of KLF8 reduced the proliferation and invasion of gastric cancer cells. Mol Biol Rep 39 9809-9815, 2012.

19. Liu L, Liu N, Xu M, Liu Y, Min J, Pang H, Zhang N, Zhang H and Zhang H: Lentivirus-delivered Krüppel-like factor 8 smal interfering RNA inhibits gastric cancer cell growth in vitro and in vivo. Tumour Biol 33: 53-61, 2012.

20. Hsu LS, Wu PR, Yeh KT, Yeh CM, Shen KH, Chen CJ and Soon MS: Positive nuclear expression of KLF8 might be correlated with shorter survival in gastric adenocarcinoma. Ann Diagn Pathol 18: 74-77, 2014.

21. Wang WF, Li J, Du LT, Wang LL, Yang YM, Liu YM, Liu H, Zhang X, Dong ZG, Zheng GX and Wang CX: Krüppel-like factor 8 overexpression is correlated with angiogenesis and poor prognosis in gastric cancer. World J Gastroenterol 19: 4309-4315, 2013.

22. Wang X, Lu H, Urvalek AM, Li T, Yu L, Lamar J, DiPersio CM, Feustel PJ and Zhao J: KLF8 promotes human breast cancer cell invasion and metastasis by transcriptional activation of MMP9. Oncogene 30: 1901-1911, 2011

23. Liu N, Wang Y, Zhou Y, Pang H, Zhou J, Qian P, Liu L and Zhang H: Krüppel-like factor 8 involved in hypoxia promotes the invasion and metastasis of gastric cancer via epithelial to mesenchymal transition. Oncol Rep 32: 2397-2404, 2014.

24. Zhang H, Liu L, Wang Y, Zhao G, Xie R, Liu C, Xiao X, Wu K, Nie Y, Zhang H and Fan D: KLF8 involves in TGF-beta-induced EMT and promotes invasion and migration in gastric cancer cells. J Cancer Res Clin Oncol 139: 1033-1042, 2013.

25. Fu WJ, Li JC, Wu XY, Yang ZB, Mo ZN, Huang JW, Xia GW, Ding Q, Liu KD and Zhu HG: Small interference RNA targeting Krüppel-like factor 8 inhibits the renal carcinoma 786-0 cells growth in vitro and in vivo. J Cancer Res Clin Oncol 136 $1255-1265,2010$.

26. Schnell O, Romagna A, Jaehnert I, Albrecht V, Eigenbrod S, Juerchott K, Kretzschmar H, Tonn JC and Schichor C: Krüppel-like factor 8 (KLF8) is expressed in gliomas of different WHO grades and is essential for tumor cell proliferation. PLoS One 7: e30429, 2012.

27. Yan Q, Zhang W, Wu Y, Wu M, Zhang M, Shi X, Zhao J, Nan Q, Chen Y, Wang L, et al: KLF8 promotes tumorigenesis, invasion and metastasis of colorectal cancer cells by transcriptional activation of FHL2. Oncotarget 6: 25402-25417, 2015.

28. Lu H, Hu L, Yu L, Wang X, Urvalek AM, Li T, Shen C, Mukherjee D, Lahiri SK, Wason MS and Zhao J: KLF8 and FAK cooperatively enrich the active MMP14 on the cell surface required for the metastatic progression of breast cancer. Oncogene 33: 2909-2917, 2014.

29. Li T, Lu H, Mukherjee D, Lahiri SK, Shen C, Yu L and Zhao J: Identification of epidermal growth factor receptor and its inhibitory microRNA141 as novel targets of Krüppel-like factor 8 in breast cancer. Oncotarget 6: 21428-21442, 2015.

30. Cao CY, Mok SW, Cheng VW and Tsui SK: The FHL2 regulation in the transcriptional circuitry of human cancers. Gene 572: $1-7,2015$.

31. Kleiber K, Strebhardt K and Martin BT: The biological relevance of FHL2 in tumour cells and its role as a putative cancer target. Anticancer Res 27: 55-61, 2007.

32. Zienert E, Eke I, Aust D and Cordes N: LIM-only protein FHL2 critically determines survival and radioresistance of pancreatic cancer cells. Cancer Lett 364: 17-24, 2015.
33. Al-Nomani L, Friedrichs J, Schüle R, Buttner R and Friedrichs N: Tumoral expression of nuclear cofactor FHL2 is associated with lymphatic metastasis in sporadic but not in HNPCC-associated colorectal cancer. Pathol Res Pract 211: 171-174, 2015.

34. Gullotti L, Czerwitzki J, Kirfel J, Propping P, Rahner N, Steinke V, Kahl P, Engel C, Schüle R, Buettner R and Friedrichs N: FHL2 expression in peritumoural fibroblasts correlates with lymphatic metastasis in sporadic but not in HNPCC-associated colon cancer. Lab Invest 91: 1695-1705, 2011.

35. Qiao L, Wang Y, Pang R, Wang J, Dai Y, Ma J, Gu Q, Li Z, Zhang Y,Zou B, et al: Oncogene functions of FHL2 are independent from NF-kappaBIalpha in gastrointestinal cancer. Pathol Oncol Res 15: 31-36, 2009.

36. Gabriel B, Fischer DC, Orlowska-Volk M, zur Hausen A, Schüle R, Müller JM and Hasenburg A: Expression of the transcriptional coregulator FHL2 in human breast cancer: A clinicopathologic study. J Soc Gynecol Investig 13: 69-75, 2006.

37. Ding L, Wang Z, Yan J, Yang X, Liu A, Qiu W, Zhu J, Han J, Zhang H, Lin J, et al: Human four- and -a-half LIM family members suppress tumor cell growth through a TGF-beta-like signaling pathway. J Clin Invest 119: 349-361, 2009.

38. Zhang W, Wang J, Zou B, Sardet C, Li J, Lam CS, Ng L, Pang R, Hung IF, Tan VP, et al: Four and a half LIM protein 2 (FHL2) negatively regulates the transcription of E-cadherin through interaction with Snail1. Eur J Cancer 47: 121-130, 2011.

39. McGrath MJ, Binge LC, Sriratana A, Wang H, Robinson PA, Pook D, Fedele CG, Brown S, Dyson JM, Cottle DL, et al: Regulation of the transcriptional coactivator FHL2 licenses activation of the androgen receptor in castrate-resistant prostate cancer. Cancer Res 73: 5066-5079, 2013.

40. He HJ, Gu XF, Xu WH, Yang DJ, Wang XM and Su Y: Krüppel-like factor 8 is a novel androgen receptor co-activator in human prostate cancer. Acta Pharmacol Sin 34: 282-288, 2013.

41. Li T, Lu H, Shen C, Lahiri SK, Wason MS, Mukherjee D, Yu L and Zhao J: Identification of epithelial stromal interaction 1 as a novel effector downstream of Kruppel-like factor 8 in breast cancer invasion and metastasis. Oncogene 33: 4746-4755, 2014.

42. Gabriel B, Mildenberger S, Weisser CW, Metzger E, Gitsch G, Schüle R and Müller JM: Focal adhesion kinase interacts with the transcriptional coactivator FHL2 and both are overexpressed in epithelial ovarian cancer. Anticancer Res 24: 921-927, 2004.

43. Wang X, Urvalek AM, Liu J and Zhao J: Activation of KLF8 transcription by focal adhesion kinase in human ovarian epithelial and cancer cells. J Biol Chem 283: 13934-13942, 2008.

44. Yi XP, Han T, Li YX, Long XY and Li WZ: Simultaneous silencing of XIAP and survivin causes partial mesenchymal-epithelial transition of human pancreatic cancer cells via the PTEN/PI3K/Akt pathway. Mol Med Rep 12: 601-608, 2015.

45. Livak KJ and Schmittgen TD: Analysis of relative gene expression data using real-time quantitative PCR and the 2(-Delta Delta $\mathrm{C}(\mathrm{T})$ ) method. Methods 25: 402-408, 2001.

46. Han T, Yi XP, Liu B, Ke MJ and Li YX: MicroRNA-145 suppresses cell proliferation, invasion and migration in pancreatic cancer cells by targeting NEDD9. Mol Med Rep 11: 4115-4120, 2015.

47. Deer EL, González-Hernández J, Coursen JD, Shea JE, Ngatia J, Scaife CL, Firpo MA and Mulvihill SJ: Phenotype and genotype of pancreatic cancer cell lines. Pancreas 39: 425-435, 2010.

48. Beuran M, Negoi I, Paun S, Ion AD, Bleotu C, Negoi RI and Hostiuc $\mathrm{S}$ : The epithelial to mesenchymal transition in pancreatic cancer: A systematic review. Pancreatology 15: 217-225, 2015.

49. Jiang JH, Liu C, Cheng H, Lu Y, Qin Y, Xu YF, Xu J, Long J, Liu L, Ni QX and Yu XJ: Epithelial-mesenchymal transition in pancreatic cancer: Is it a clinically significant factor. Biochim Biophys Acta 1855: 43-49, 2015.

50. Yi X, Li Y, Zai H, Long X and Li W: KLF8 knockdown triggered growth inhibition and induced cell phase arrest in human pancreatic cancer cells. Gene 585: 22-27, 2016.

51. Thiery JP, Acloque H, Huang RY and Nieto MA: Epithelial-mesenchymal transitions in development and disease. Cell 139: 871-890, 2009. 